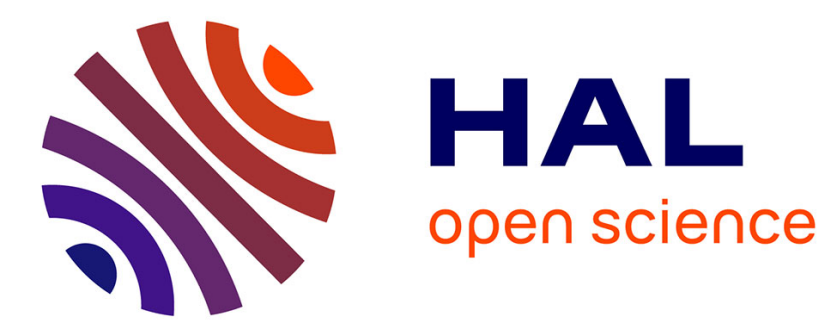

\title{
Beat-Wave Experiments in the Micro Wave Range: Pump Depletion
}

N. Andreev, E. Campos, B. Cros, J. Godiot, L. Gorbunov, G. Matthieussent

\section{To cite this version:}

N. Andreev, E. Campos, B. Cros, J. Godiot, L. Gorbunov, et al.. Beat-Wave Experiments in the Micro Wave Range: Pump Depletion. Journal de Physique IV Proceedings, 1995, 05 (C6), pp.C6-75-C6-78. 10.1051/jp4:1995614 . jpa-00253977

\section{HAL Id: jpa-00253977 https://hal.science/jpa-00253977}

Submitted on 1 Jan 1995

HAL is a multi-disciplinary open access archive for the deposit and dissemination of scientific research documents, whether they are published or not. The documents may come from teaching and research institutions in France or abroad, or from public or private research centers.
L'archive ouverte pluridisciplinaire HAL, est destinée au dépôt et à la diffusion de documents scientifiques de niveau recherche, publiés ou non, émanant des établissements d'enseignement et de recherche français ou étrangers, des laboratoires publics ou privés. 


\title{
Beat-Wave Experiments in the Micro Wave Range: Pump Depletion
}

\author{
N.E. Andreev, E.D. Campos*, B. Cros*, J. Godiot*, L.M. Gorbunov** and G. Matthieussent* \\ Plasma Theory Division, Institute for High Temperatures of RAS, Izhorskaya St. 12/19, \\ Moscow, 127412, Russia \\ * Laboratoire de Physique des Gaz et des Plasmas, Université Paris XI-CNRS, \\ 91405 Orsay cedex, France \\ ** Plasma Theory Division, Lebedev Physical Institute of RAS, Leninsky pr. 53, Moscow, \\ 117924, Russia
}

\begin{abstract}
Excitation of electron plasma waves by the mixing of two microwave beams is studied in a laboratory plasma. The first experimental observation of pump wave depletion is presented. The numerical calculations, based on one-dimensional model, describing space-time evolution of eight interacting waves, are in good agreement with experimental data. Estimation of the depletion on the basis of conservation laws is also discussed.
\end{abstract}

\section{INTRODUCTION}

The nonlinear excitation of plasma waves by beating between two electromagnetic waves has a wide range of applications in plasma physics, including plasma heating $[1,2,3]$, plasma diagnostics $[4,5]$, ionospheric plasma studies and modification ${ }^{[6]}$, acceleration of electrons ${ }^{[7,8]}$. Main attention is focused on the amplitude of the excited plasma waves ${ }^{[9]}$. The variation of pump waves is usualy neglected although energy is partially transferred from one electromagnetic wave with higher frequency $\omega_{1}$ to the other with lower frequency $\omega_{2}$ and plasma oscillations with frequency, $\omega=\omega_{1}-\omega_{2}$, near electron plasma frequency $\omega_{\mathrm{p}}$. As a result, the higher frequency wave loses energy and therefore is depleted.

We present here the first experimental observation of pump wave depletion appearing during resonant plasma wave excitation by nonlinear mixing of two electromagnetic waves. A simplified theoretical model describing the space-time evolution of both electromagnetic and plasma waves is in good agreement with experimental data. We also derive approximate expressions for the amplitudes of plasma waves and for the depletion of the higher-frequency pump wave.

The experiment is performed in a large volume $\left(\sim \mathbf{0 , 8} \mathbf{m}^{\mathbf{3}}\right)$ of unmagnetized plasma, created in a cylindrical multipolar discharge ${ }^{[10]}$ with following parameters: electron density $\mathrm{n}_{\mathbf{0}} \simeq 1,7.10^{11} \mathrm{~cm}^{-3}$, neutral (Ar) density $\sim 10^{13} \mathrm{~cm}^{-3}$, electron temperature $T_{e} \simeq 3 \mathrm{eV}$, electron-ion collision frequency $\nu_{\mathrm{ei}} \simeq 10^{6} \mathrm{~s}^{-1}$, electron-neutral collision frequency $\nu_{\mathrm{ea}} \simeq 10^{6} \mathrm{~s}^{-1}$. The electromagnetic waves are launched in the chamber by small horns (Fig. 1). One of them, with lower frequency $5,6 \mathrm{GHz}\left(\omega_{2}=3,53.10^{10} \mathrm{~s}^{-1}\right)$ and pulse duration $\tau_{2}=1,25 \mu \mathrm{s}$, is introduced along the chamber axis. The other wave with higher frequency $9,3 \mathbf{~ G H z}\left(\omega_{1}=5,86.10^{10} \mathrm{~s}^{-1}\right)$ has a pulse duration $\tau_{1}=1,5 \mu \mathrm{s}$ and is injected at $30^{\circ}$ inclination with respect to the axis of the chamber. The peak power for both waves is near $200 \mathrm{~kW}$.

The amplitude of the electric fields of both electromagnetic and plasma waves, as well as the electron density are measured with a small cylindrical Langmuir probe ( $\phi \simeq 0,4 \mathrm{~mm}$, length

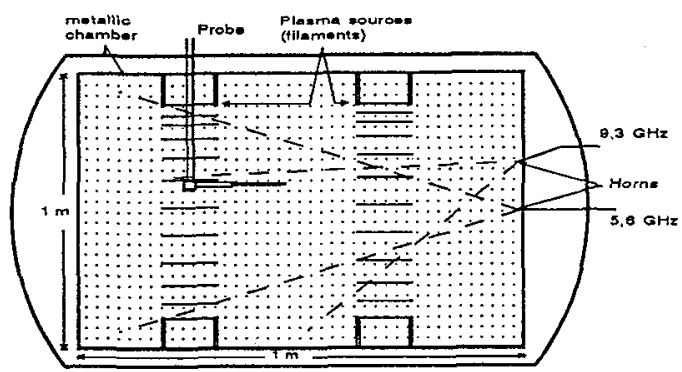

Figure 1: Schematic diagram of the beat-wave experiment. Dotted lines indicate the boundaries of the beams.

$\simeq 2,0 \mathrm{~mm}$ ) situated at a distance $-40 \mathrm{~cm}$ from side wall and at a distance $\sim 50 \mathrm{~cm}$ from front wall of the chamber. The measurements of electron density are done approximately $1 \mu \mathrm{S}$ before the high frequency pulse. For this the probe is biased at a potential $+10,5 \mathrm{~V}$ above the plasma potential. The high frequency fields in the plasma are detected by a non polarized probe. The signal is splitted and then the use of narrow frequency filters, followed by quadratic detectors, allows to measure separatly the amplitude 
of the electric field of each wave.

The absolute value of the electric fields of electromagnetic waves in the plasma is estimated on the basis of ion-sound waves excitation by the action of ponderomotive forces produced by a standing electromagnetic wave ${ }^{[11]}$. As result we evaluate the amplitudes of both $\mathbf{h f}$ waves as $\mathbf{E}_{10} \sim \mathbf{E}_{20} \sim 4 \div$ $6.10^{4} \mathrm{~V} / \mathrm{m}$ and the effective coefficient of reflection from the walls of the chamber is approximately measured to be $\mathbf{r} \sim \mathbf{0 , 6}$.

Electron density variations, of the order of $10^{-3}$ in relative value, with temporal scale of the order of $5.10^{-3} \mathrm{~s}$, arise due to the residual modulation of the power supplies of the multipolar discharge.

The amplitude of the detected plasma oscillations shows a sharp resonant dependence with electron density. Among the measurements of plasma waves amplitude with frequency $\omega=\omega_{1}-\omega_{2}=2,3.10^{10} s^{-1}$ we take the one with maximum amplitude. We suppose that the electron density in this case is the resonant one. Normalizing the amplitude of plasma oscillations as well as plasma density to these resonant values, we obtain the points shown in Fig.2. For comparison Fig.2 shows also the theoretical curve obtained when plasma wave saturation is due to resonant density detuning. The maximum plasma wave amplitude is taken to be determined by the driving pulse duration $1 \mu \mathrm{S}$. It is seen that the halfwidth of the curve corresponds to a density change of $0,05 \%$ in relative value. However, the experimental incertitude in determination of both the plasma density and the plasma wave amplitude doesn't allow to speak of an agreement between experimental results and theoretical prediction. Nevertheless, a strong resonant dependence of plasma wave amplitude on electron density is seen.

The temporal variation of the square of electric field both for microwave pulses and for plasma wave are shown in Fig. 3 in the case of resonant density. The higher-frequency pulse begins approximately $0,2 \mu \mathrm{s}$ later and is completed $0,4 \mu \mathrm{s}$ later as compared to the lower-frequency pulse. The plasma oscillations are excited only during the overlapping of pulses. The depletion of the higherfrequency wave is observed also only at this time. After turn off of the lower-frequency wave, the higher-frequency wave restors its amplitude. The depletion of the higher-frequency wave takes place only if the electron density is near to the resonant value and if the amplitude of plasma oscillations is

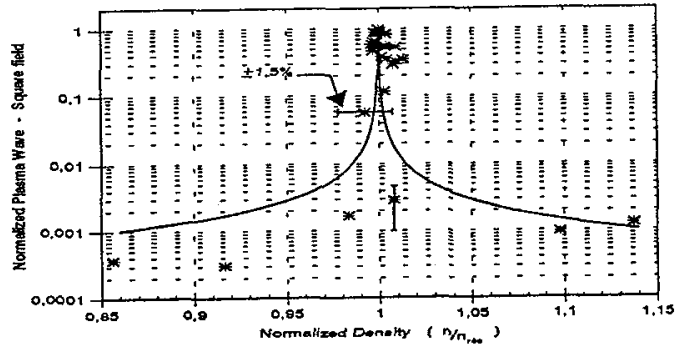

Figure 2: Dependence of the normalized amplitude of plasma wave on the normalized plasma density.

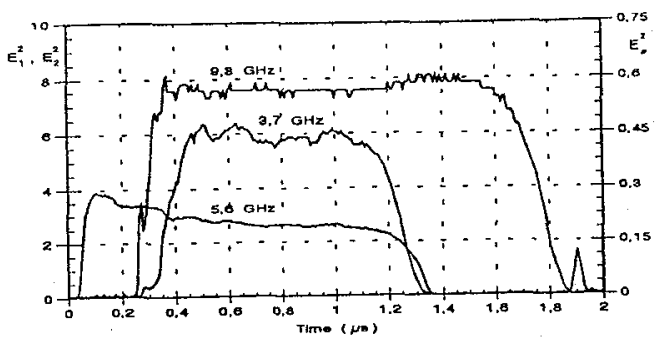

Figure 3: Temporal variation of the square of microwave $\left(E_{1}^{2}\right.$. $\left.E_{2}^{2}\right)$ and plasma $\left(E_{1}^{2}\right)$ electric fields in arbitrary units. sufficiently high.

In order to treat the experimental results we examine a one-dimensional theoretical model describing the space-time evolution of eight coupled waves in a uniform plasma of finite lenght $\mathbf{L}$. The subset of electromagnetic waves contains a pair of right-going waves $\left(\mathbf{E}_{1}\right.$ and $\left.\mathbf{E}_{2}\right)$ with frequencies $\omega_{1}$ and $\omega_{2}$ and with corresponding wave numbers $\mathbf{k}_{1}$ and $\mathbf{k}_{2}$, and a pair of reflected left-going waves $\left(\mathbf{E}_{3}\right.$ and $\left.\mathbf{E}_{4}\right)$ with the same frequencies and opposite wave number. These waves interact with four plasma waves of equal frequency $\omega=\omega_{1}-\omega_{2}$ but with different wavelengths. One pair of conterpropagating plasma waves ( $\mathbf{E}_{\mathrm{p} 1}$ and $\mathbf{E}_{\mathrm{p} 3}$ ) has the wave number $\mathbf{k}=\mathbf{k}_{1}-\mathbf{k}_{2}$ (long waves) as the other one ( $\mathbf{E}_{\mathrm{p2}}$ and $\mathbf{E}_{\mathrm{p} 4}$ ) has the wave number $\mathbf{K}=\mathbf{k}_{\mathbf{1}}+\mathbf{k}_{\mathbf{2}}$ (short waves).

The set of equations for the slowly varying amplitudes of electromagnetic waves may be obtained by the usual method (see, for example, [2]) and has the form

$\left(\frac{\partial}{\partial t}+v_{1} \frac{\partial}{\partial x}\right) E_{1}=-i\left(\frac{\omega_{D}^{2}}{4 \omega_{2}}\right)\left(E_{2} q_{1}+E_{4} q_{2}\right)$.

$$
\left(\frac{\partial}{\partial t}+v_{2} \frac{\partial}{\partial x}\right) E_{2}=-i\left(\frac{\omega_{p}^{2}}{4 \omega_{1}}\right)\left(E_{1} q_{1}^{*}+E_{3} q_{4}^{*}\right)
$$


$\left(\frac{\partial}{\partial t}-v_{1} \frac{\partial}{\partial x}\right) E_{3}=-i\left(\frac{\omega_{p}^{2}}{4 \omega_{2}}\right)\left(E_{4} q_{3}+E_{2} q_{4}\right)$

$$
\left(\frac{\partial}{\partial t}-v_{2} \frac{\partial}{\partial x}\right) E_{4}=-i\left(\frac{\omega_{p}^{2}}{4 \omega_{1}}\right)\left(E_{3} q_{3}^{*}+E_{1} q_{2}^{*}\right)
$$

where $\nabla_{1,2}=c^{2} k_{1,2} / \omega_{1,2}$ are the group velocities of electromagnetic waves with frequencies $\omega_{1}$ and $\omega_{2}$ respectively, $q_{i}$ is the slowly varying amplitude of the electron density perturbation for the corresponding plasma wave

$$
q_{1}=\frac{i K E_{p 1}}{4 \pi n_{0} e}, q_{3}=-\frac{i k E_{p 3}}{4 \pi n_{0} e}, q_{2}=\frac{i K E_{p 2}}{4 \pi n_{0} e}, q_{4}=-\frac{i K E_{p 4}}{4 \pi n_{0} e}
$$

The set of equations for $q_{i}$, taking under consideration the nonlinearity due to relativistic electron mass variation $^{[12]}$, the collisional damping and the frequency mismatch $\Delta \simeq\left(\omega^{2}-\omega_{p}^{2}\right) / 2 \omega^{2}$, has the form

$\left(\frac{i}{\omega} \frac{\partial}{\partial t}+\Delta+i \gamma+\frac{3}{16}\left|q_{1}\right|^{2}\right) q_{1}=\alpha k^{2} E_{1} E_{2}^{*}$

$\left(\frac{i}{\omega} \frac{\partial}{\partial t}+\Delta+i \gamma+\frac{3}{16}\left|q_{3}\right|^{2}\right) q_{3}=\alpha K^{2} E_{1} E_{4}^{*}$,

$$
\begin{aligned}
& \left(\frac{i}{\omega} \frac{\partial}{\partial t}+\Delta+i \gamma+\frac{3}{16}\left|g_{2}\right|^{2}\right) q_{2}=\alpha k^{2} E_{3} E_{4}^{*},(7) \\
& \left(\frac{i}{\omega} \frac{\partial}{\partial t}+\Delta+i \gamma+\frac{3}{16}\left|q_{4}\right|^{2}\right) g_{4}=\alpha K^{2} E_{3} E_{2}^{*},(9)
\end{aligned}
$$

where $\gamma=\left(\nu_{\mathrm{ei}}+\nu_{\mathrm{ea}}\right) / \omega, \alpha=\mathrm{e}^{2} / 4 \mathrm{~m}^{2} \omega_{1} \omega_{2} \omega^{2}$

To solve this set of coupled equations, (1)-(4) and (6)-(9), we suppose that in initial moment of time both electromagnetic and plasma waves are absent everywhere in region $0 \leq x \leq L$. The two electromagnetic pulses $\mathbf{E}_{1 \mathrm{i}}(\mathbf{t})$ and $\mathbf{E}_{2 \mathrm{i}}(\mathbf{t})$ are introduced in a plasma layer through the boundary $\mathbf{x}=\mathbf{0}$ with some delay one with respect to another. The temporal variation of the amplitudes are taken to be in accordance with experimental data.

At the boundary $x=0$ the conditions $E_{1}(t)=E_{1 i}(t)+r E_{3}(t)$ and $E_{2}(t)=E_{2 i}(t)+r E_{4}(t)$ are supposed to be valid. At the other boundary $x=L$ we assume $\mathbf{r E}_{1} \exp \left(\mathbf{i k}_{1} L\right)=E_{3} \exp \left(-\mathbf{i k}_{1} L\right)$ and $r E_{2} \exp \left(i_{2} L\right)$ $=\mathbf{E}_{\mathbf{4}} \exp \left(-\mathrm{ik} \mathrm{k}_{2} \mathrm{~L}\right.$ ) where the reflection coefficient $\mathbf{r}$ is taken to be real and the same for both electromangetic waves.

To model the experimental conditions we assume in our numerical calculations that the thickness of the plasma is $\mathbf{L}=80 \mathrm{~cm}$, the coefficient of reflection $\mathbf{r}=0,5, \omega_{1}=6.10^{10} \mathrm{~s}^{-1}, \omega_{2}=3,5.10^{10} \mathrm{~s}^{-1}, \mathbf{E}_{10}=\mathbf{E}_{20}$, $\mathbf{t}_{0}=1 \mu \mathrm{s}$. Fig. 4 shows the temporal variation of the normalized wave-energy density at frequencies $\omega_{1}$ (dark line), $\omega_{2}$ (dotted line) and $\omega=\omega_{1}-\omega_{2}$ (dashed line) calculated at the point $x=41 \mathrm{~cm}$. All values are given in units of the peak incident energy density $\mathbf{E}_{10}{ }^{2} / 8 \pi$ where the ratio of the electron quiver velocity to the light velocity $\left(v_{10} / c\right)$, corresponding to the maximum of amplitude $E_{1 i}(t)$, is choosen to be $7,5.10^{-4}$. The mismatch and damping rate are taken to be $\Delta=\mathbf{2 . 1 0 ^ { - 4 }}$ and $\gamma=3.10^{-4}$.

The comparison of experimental results in Fig. 3 with the results of a numerical simulation (Fig.4) allows to speak of a good agreement between them.

Having in mind the smallness of the depletion effect we are able to formulate simplified considerations on Eqs. (1) - (9). The amplitudes of plasma waves, $\mathbf{E}_{\mathrm{pj}}$, may be in quasistatic approximation ( $\partial / \partial t\langle\nu$, $\omega_{\mathrm{p}} \Delta$ ) calculated by means of Eqs. (0) - (9) in which the amplitudes of electromagnetic waves $\mathbf{E}_{\mathbf{j}}$ are determined by Eqs. (1) - (4) with right hand sides equal to zero. As the result we find

$$
\sum_{j=1}^{4}\left|E_{p j}\right|^{2}=\left(\frac{e}{4 m \omega_{1} \omega_{2}}\right)^{2} \frac{\left|E_{i 0}\right|^{2}\left|E_{20}^{\prime}\right|^{2}}{\Delta^{2}+\gamma^{2}}\left[k^{2}\left(1+I^{4}\right)+2 I^{2} K^{2}\right]
$$

where the values $\left|E_{10,20}^{\prime}\right|^{2}=\left|E_{10,20}\right|^{2}\left[1+r^{4}-2 r^{2} \cos \left(2 k_{1,2} L\right]^{-1}\right.$ determine the amplitudes of the electromagnetic waves inside the plasma taking into account the reflection from walls effects. With our experimental parameters the last term in square brackets of expression (10) is the major one and it is due to the short plasma wave.

The Eqs. (1) - (4) and (6) - (9) allow us to find the conservation laws for the transverse action (ManleyRowe, or photon conservation) and for the energy (with dissipation) by means of a method analogeous to the one used in paper ${ }^{[2]}$. In stationary case, when the amplitudes of electromagnetic and plasma waves don't change in time, these conservation laws give 


$$
\frac{v_{1}}{\omega_{1}} \frac{\partial}{\partial x}\left(\left|E_{1}\right|^{2}-\left|E_{3}\right|^{2}\right)=-2 \gamma \sum_{j=1}^{4}\left|E_{p j}\right|^{2}
$$

The

Eq.(11) denotes that the spatial variation in higher frequency photon flux is equal to total dissipation rate of plasmons. Upon integrating Eq.(11) over $\mathbf{x}$ on the length of the plasma we find the full reduction of the square amplitude of the higherfrequency wave during its propagation from $x=0$ to $\mathbf{x}=\mathbf{L}$ and back

$$
\Delta|E|^{2}=\left(\left|E_{1}(0)\right|^{2}-\left|E_{1}(L)\right|^{2}\right)+\left(\left|E_{3}(L)\right|^{2}-\left|E_{3}(0)\right|^{2}\right. \text {, }
$$

$$
\Delta|E|^{2}=2 \gamma L \frac{\omega_{1}}{V_{1}} \sum_{j=1}^{4}\left|E_{p j}\right|^{2}
$$

The value $\Delta|\mathbf{E}|^{2}$ may be used as an estimation of the wave depletion discussed above. Substituting in Eq. (12) the expression (10) and using the indicated above set of number we obtain $\left(\Delta|E|^{2} /\left|E_{10}\right|^{2}\right) \times 5 \cdot 10^{-2}$ which is in quite good agreement with results of the numerical simulations and of the experiment.

The observation of the pump-wave depletion requires two conditions. From one side, the pump depletion begins to be important, in the resonant conditions, when the plasma wave has a rather high amplitude. From the other side, for a to high amplitude, the intense plasma wave undergoes the parametric instabilities ${ }^{[13]}$ and its regulare structure is destroied. Hence, the effect of pump-wave depletion is allowed to be observed in a sufficiently narrow range of parameters.

\section{References}

[1] - M.N.Rosenbluth and C.S.Liu, Phys. Rev. Lett., 29, 701 (1972)

[2] - B.Cohen, A.Kaufman and K.Watson, Phys. Rev, Lett., 29, 581 (1972)

[3] - B.Cohen, Phys. of Fluids, 17, 496 (1974); B.Cohen et al., Phys. of Fluids, 18, 470 (1975); V.Fuchs et al., Phys. Rev. Lett., 31, 1110 (1973)

[4] - N.Kroll, A.Ron and N.Rostoker, Phys. Rev. Lett., 13, 83 (1964)

[5] - R.Cano, I.Fidone and B.Zanfagna, Phys. of Fluids, 14, 811 (1971); E.S.Weibel, Phys. Rev. Lett., 37, 1619 (1976); B.Stansfield, R.Nodwell, Phys. Rev. Lett., 26, 1219 (1971); L.A.Godfrey, Phys. Rev. A, 20, 567 (1975)

[6] - J.Lavergnat et al., Geoph. Res. Lett., 4, 417 (1977); K.Rypdal, J. Geophys., 86, 1544 (1981); V.A.Puchkov, J. Plasma Phys., 9, 718 (1983); G.Weil, Phys. Fluids, 13, 1802 (1970)

[7] - T.Tajima and J.M.Dawson, Phys. Rev. Lett., 43, 267 (1979)

[8] - C.E.Clayton et al., Phys. Plasma, 1, 1753 (1994); F.Amiranoff et al., Phys. Rev. Lett., 68, 48 (1992); Y.Kitagawa et al., Phys. Rev. Lett., 68, 48 (1992); N.A.Ebrahim, J. Appl. Phys., 76, 7645 (1994) [9] - B.Amini, Phys. Rev. Lett., 54, 1163 (1985); P.S.Lee et al., Phys. Rev. Lett., 30, 538 (1973); G.Schmidt, Phys. Fluids, 16, 1676 (1973); B.Amini et al., Phys. Rev. Lett., 53, 1441 (1984); B.Amini, Phys. Fluids, 28, 387 (1985); C.M.Tang, P.Sprangle and R.N.Sudan, Appl. Phys. Lett., 45, 375 (1984) [10] - M.Benhassine et al., Rev. Phys. Appl., 19, 545 (1984)

[11] - P.Fischer et al., J. Phys., 48, 233 (1987)

[12] - C.J.McKinstrie and D.W.Forslund, Phys. Fluids, 30, 904 (1987)

[13] - Ya.L.Bogomolov et al., Physica Scripta, 52, 51 (1994) 